\title{
AVALIAÇÃO DE VARIEDADES DE MORANGUEIRO EM SISTEMAS HIDROPÔNICOS SOB CASA DE VEGETAÇÃ̃ ${ }^{1}$
}

\author{
EDILSON COSTA ${ }^{2} \&$ PAULO MARTINS LEAL ${ }^{3}$
}

RESUMO-Realizaram-se experimentos na Faculdade de Engenharia Agrícola na Universidade Estadual de Campinas, com quatro variedades de morangueiro (Campinas, Seascape, Sweet Charlie e Tudla), em quatro sistemas de produção hidropônica (canal de $100 \mathrm{~mm}$, canal de $150 \mathrm{~mm}$, canal de $150 \mathrm{~mm}$ com vaso contendo fibra de coco e tubo vertical contendo casca de arroz carbonizada), em três ambientes protegidos com níveis tecnológicos diferenciados (casa de vegetação sem resfriamento evaporativo do ar e sem injeção aérea de $\mathrm{CO}_{2}$, casa de vegetação com injeção aérea de $\mathrm{CO}_{2}$ e sem resfriamento evaporativo do ar, e casa de vegetação com injeção aérea de $\mathrm{CO}_{2}$ e resfriamento evaporativo do ar). Foram analisadas as produtividades em gramas por planta (P) e o número de frutos por planta (NF). Destacou-se como melhor variedade a Campinas. O melhor sistema de cultivo foi o de canais de $150 \mathrm{~mm}$ com vaso contendo fibra de coco.

Termos para indexação: Fragaria ananassa Duch; Hidroponia; Estufas.

\section{EVALUATION OF STRAWBERRY VARIETIES IN HYDROPONICS SYSTEMS IN GREENHOUSES}

ABSTRACT- Experiments were accomplished in the experimental field of the Faculty of Agricultural Engineering at State University of Campinas (Unicamp). Four varieties of strawberry were tested (Campinas, Seascape, Sweet Charlie and Tudla) in four hydroponics production systems $(100 \mathrm{~mm}$ and $150 \mathrm{~mm}$ channels and $150 \mathrm{~mm}$ channel with a vase containing coconut fiber and a vertical pipe containing carbonized rice skin) in three greenhouses with different technical: i) greenhouse without air evaporative cooling nor $\mathrm{CO}_{2}$ aerial injection, ii) greenhouse with $\mathrm{CO}_{2}$ aerial injection and without air evaporative cooling and iii) greenhouse with $\mathrm{CO}_{2}$ aerial injection and air evaporative cooling. The productivity in grams per plant (P) and the number of fruits per plant (NF) were analyzed. The variety Campinas was better than the others. The best hydroponics system was the $150 \mathrm{~mm}$ channel with a vase containing coconut fiber.

Index terms: Fragaria ananassa Duch, Hydroponics, Greenhouses.

\section{INTRODUÇ̃̃O}

O desenvolvimento do morangueiro em sistemas hidropônicos vem apresentando resultados promissores em pesquisas desenvolvidas nas regiões Sul e Sudeste brasileiras. No entanto, constata-se, nas regiões produtoras, um número muito reduzido de produções que estejam utilizando os sistemas hidropônicos para exploração dessa hortaliça de fruto, fato que pode ser explicado pelo alto investimento inicial que o sistema exige.

Segundo Nunes 2000, citado por Carrijo et al. (2004), a utilização de fibra de coco como substrato, em produções fora do solo, promove um excelente desenvolvimento à planta, principalmente por reter maior quantidade de umidade e propiciar melhor desenvolvimento do sistema radicular.

Gusmão (2000), na região de Jaboticabal-SP, avaliando duas variedades de morangueiro, Campinas e Seascape, em sistema hidropônico NFT (Nutrient Film Technique), com canais de $100 \mathrm{~mm}$, em ambiente protegido, observou que a variedade Campinas apresentou produção, crescimento vegetativo e emissão de estolhos maior que a variedade Seascape.

Tavares (2001), estudando o morangueiro em cultivo hidropônico no Distrito Federal, obteve para a produção de quatro cultivares de morangueiro, Campinas, Dover, Seascape e Toyonoka, a produtividade por plantas, de 406,6g; 268,3g; 136,1g e $162,1 \mathrm{~g}$, respectivamente. Ou seja, a variedade Campinas, também, se destacou nesse experimento.

A produção do morangueiro em sistema hidropônico vertical (coluna de cultivo), porém, é menor que a produção no sistema de bancadas horizontais com canais de $100 \mathrm{~mm}$; existe melhor aproveitamento do espaço interno do ambiente protegido, proporcionando reflexos positivos no rendimento por área, ou seja, na produtividade (Fernandes Júnior, 2001).

A exploração dessa hortaliça de fruto veio sempre acompanhada pela grande quantidade de defensivos químicos, $\mathrm{e}$, no intuito de minimizar esse agravante, surgiram alternativas promissoras de exploração em ambientes protegidos (Goto \& Tivelli, 1998), procurando minimizar a ocorrência de doenças (Pires et al., 1999).

O objetivo deste trabalho foi avaliar a produtividade e o desenvolvimento de quatro cultivares de morangueiro (Campinas, Seascape, Sweet Charlie e Tudla) nos sistemas hidropônicos de canais de $100 \mathrm{~mm}$, canais de $150 \mathrm{~mm}$, canais de $150 \mathrm{~mm}$ com vasos contendo fibra de coco e coluna vertical com casca de arroz carbonizada, em três tipos de ambientes protegidos.

1(Trabalho 138-07). Recebido em: 31-05-2007. Aceito para publicação em: 17-12-2007.Parte da Tese de Doutorado do primeiro autor.

2Professor, UEMS/Aquidauana, Rodovia Aquidauana/CEPA, km 12, Aquidauana - MS, CEP: 79.200-000. E-mail:mestrine@uems.br.

${ }^{3}$ Professor, FEAGRI-UNICAMP, Campinas - SP. E-mail: pamleal@agr.unicamp.br. 


\section{METODOLOGIA}

No período de abril de 2002 a março de 2003, foram desenvolvidos experimentos com o cultivo hidropônico do morangueiro, em três casas de vegetação da Faculdade de Engenharia Agrícola - FEAGRI/UNICAMP, utilizando as variedades Campinas, Seascape, Sweet Charlie e Tudla, nos sistemas de cultivo de bancadas tipo "A-frame" (Seção A) com a técnica da solução do filme nutriente (NFT) e sistema de tubos verticais com substrato. $\mathrm{O}$ ambiente 1 foi uma casa de vegetação que não teve injeção de $\mathrm{CO}_{2}$ sem resfriamento evaporativo do ar. $\mathrm{O}$ ambiente 2 foi uma casa de vegetação que teve injeção de $\mathrm{CO}_{2}$ sem resfriamento evaporativo do ar e o ambiente 3 foi uma casa de vegetação que possuía injeção de $\mathrm{CO}_{2}$ e resfriamento evaporativo do ar.

Os sistemas hidropônicos utilizados foram assim designados: Sistema 1 - Bancada com 12 canais de $100 \mathrm{~mm}$ em NFT; Sistema 2 - Bancada com 6 canais de 150mm em NFT; Sistema 3 - Bancada com 6 canais de 150mm em NFT, com vaso contendo substrato fibra de coco; Sistema 4 - Tubos verticais contendo 4 linhas com sete plantas cada, totalizando 28 plantas por tubo. Os tubos foram preenchidos com casca de arroz carbonizada. Todos os sistemas de bancadas continham 15 plantas por canal de cultivo.

As variedades de morangueiro foram designadas, também, por número, onde 1 (Campinas), 2 (Seascape), 3 (Sweet Charlie) e 4 (Tudla). A variedade Campinas é de origem brasileira, obtida no Instituto Agronômico de Campinas, em 1960; a variedade Sweet Charlie é de origem norte-americana, obtida na Universidade da Flórida, em 1992, e a variedade Tudla é de origem espanhola, lançada em 1992 (Oliveira et al., 2005). A variedade Seascape é de origem norte-americana, obtida na Universidade da Califórnia, em 1991 (Bernardi et al., 2005).

Utilizou-se a solução nutritiva recomendada por Fernandes Júnior et al. (2001), sendo que a mesma foi refrigerada por um sistema de expansão.

$\mathrm{O}$ dióxido de carbono $\left(\mathrm{CO}_{2}\right)$, injetado diretamente às folhas das plantas, foi aplicado em três períodos: das $9 \mathrm{~h} 30$ às $10 \mathrm{~h}$, das $10 \mathrm{~h} 15$ às $10 \mathrm{~h} 45 \mathrm{e}$ das $11 \mathrm{~h}$ às $11 \mathrm{~h} 30$. No ambiente 2 , quando a temperatura permitia, $\mathrm{o} \mathrm{CO}_{2}$ era aplicado com as cortinas laterais fechadas.

As cortinas internas, termorefletoras com $60 \%$ de sombra, possuíam abertura e fechamento realizados por motores reversos, através de controlador com leitura de um fotossensor LDR (Light Dependent Resistor) ou RVL (Resistor Variável de Luz).

O sistema de resfriamento evaporativo instalado no Ambiente 3 foi composto pelo meio poroso de celulose e dois exaustores que exauriam o ar interno.

No experimento, a solução nutritiva foi refrigerada, sendo que, nos horários mais quentes dos dias de primavera e verão, sua temperatura não ultrapassou os $23^{\circ} \mathrm{C}$. Os autores Villela Júnior et al. (2004), em experimentos com morangueiro hidropônico, destacaram o efeito benéfico do resfriamento da solução nutritiva para o desenvolvimento da planta.

Para análise de variância, foi utilizado o delineamento inteiramente casualizado, no esquema de parcelas subdivididas, para cada ambiente de cultivo, onde as parcelas principais foram os sistemas hidropônicos, e as subparcelas foram as variedades do morangueiro. Utilizaram-se 5 (cinco) repetições. As variáveisresposta foram produtividade em grama por planta $(\mathrm{P})$ e número de frutos por planta (NF).

Os dados foram submetidos à análise de variância, e as médias, ao teste de Tukey, no nível de $5 \%$ de probabilidade. As análises foram realizadas pelo programa computacional ESTAT (Sistema de Análise Estatística - UNESP/FCAVJ, 1994).

\section{RESULTADOS E DISCUSSÃO}

As análises de variância para as variáveis Produtividade (P) e Número de Frutos (NF) demonstraram que as parcelas, subparcelas e interações apresentaram diferenças significativas.

Na Tabela 1, para o ambiente 1, pode-se observar que o melhor sistema hidropônico de produção foi o sistema de $150 \mathrm{~mm}$ com vaso contendo fibra de coco (sistema 3), tanto para a produtividade como para o número de frutos. Resultado que vem de acordo com o comentário de Nunes 2000, citado por Carrijo et al. (2004) a respeito da utilização da fibra de coco no melhor desenvolvimento radicular da planta. $\mathrm{Na}$ avaliação dos sistemas, verifica-se, ainda que, para o número de frutos, os demais sistemas não diferiram. No entanto, para a produtividade, os sistemas 1 e 2 propiciaram maior produtividade que o sistema 4 .

Ainda na Tabela 1, avaliando as variedades, observa-se que a variedade Campinas (variedade 1) foi a que demonstrou melhor desempenho, apresentando maior produtividade e maior número de frutos, fato também observado pelos autores Gusmão (2000) e Tavares (2001) em experimentos com morangueiro hidropônico. Verifica-se, ainda, que a variedade Sweet Charlie (variedade 3) apresentou maior número de frutos que as variedades Seascape e Tudla (variedades 2 e 4, respectivamente). A variedade 4 propiciou maior produtividade que a variedade 2 .

Nas Tabelas 2 e 3, estão apresentados os testes de Tukey para comparação de médias dos desdobramentos das interações, para o ambiente 1, em nível de $5 \%$ de probabilidade.

De acordo com a Tabela 2, onde está apresentado o desdobramento das variedades dentro dos sistemas de produção, observa-se que, para o sistema 1, a variedade Campinas, que não diferiu significativamente da variedade Tudla, apresentou maior produtividade que as variedades Sweet Charlie e Seascape, apresentando, também, maior número de frutos que as demais variedades. Para os sistemas 2 e 3, a variedade Campinas foi a que apresentou maior média de produtividade e número de frutos. Para o sistema 4, a variedade Campinas, que não diferiu significativamente da variedade Seascape, apresentou maior produtividade que a variedade Tudla, apresentando, também, maior número de frutos que as demais variedades.

Analisando o desdobramento de sistemas dentro das variedades, (Tabela 3), avaliando a média de produtividade e de número de frutos dos sistemas dentro de cada variedade, separadamente, observa-se que, para todas as variedades, o sistema de $150 \mathrm{~mm}$ com vaso contendo fibra de coco (sistema 3 ) apresentou o melhor desenvolvimento das plantas, tanto para a produtividade como para o número de frutos. 
Avaliando a Tabela 4, no ambiente 2, pode-se observar que o melhor sistema hidropônico de produção foi o sistema de $150 \mathrm{~mm}$ com vaso contendo fibra de coco (sistema 3 ), tanto para a produtividade como para o número de frutos. Resultado encontrado, também, para a avaliação no ambiente 1, destacando esse sistema de produção e reforçando o comentário de Nunes 2000, citado por Carrijo et al. (2004) a respeito da utilização da fibra de coco.

Ainda na Tabela 4, avaliando as variedades, observa-se que a variedade Campinas (variedade 1) foi a que apresentou melhor desempenho, apresentando maior produtividade e maior número de frutos, fato também observado no ambiente 1 , reafirmando os resultados encontrados pelos autores Gusmão (2000) e Tavares (2001) em experimentos com morangueiro hidropônico, conforme destacado anteriormente.

Nas Tabelas 5 e 6, estão apresentados os testes de Tukey para comparação de médias dos desdobramentos das interações, no ambiente 2 , em nível de $5 \%$ de probabilidade.

De acordo com a Tabela 5, onde está apresentado o desdobramento das variedades dentro dos sistemas de produção, observa-se que, para os sistemas 1 e 2, a variedade Campinas foi a que apresentou maior média de produtividade e número de frutos. Para o sistema 3, a variedade Campinas, que não diferiu significativamente da variedade Tudla, apresentou maior produtividade que as variedades Sweet Charlie e Seascape, apresentando, também, maior número de frutos que todas as variedades estudadas. Para o sistema 4, verifica-se que a variedade Tudla apresentou desenvolvimento inferior ao das variedades Sweet Charlie e Campinas.

Analisando o desdobramento de sistemas dentro das variedades, (Tabela 6), avaliando a média de produtividade e de número de frutos dos sistemas dentro de cada variedade, separadamente, observa-se que, para todas as variedades, o sistema de $150 \mathrm{~mm}$ com vaso contendo fibra de coco (sistema 3 ) apresentou o melhor desenvolvimento das plantas, tanto para a produtividade como para o número de frutos.

Na Tabela 7, no ambiente 3, pode-se observar que o melhor sistema hidropônico de produção foi o sistema de $150 \mathrm{~mm}$ com vaso contendo fibra de coco (sistema 3 ), tanto para a produtividade como para o número de frutos. Esse resultado, também, foi encontrado nos ambientes 1 e 2 .

Ainda na Tabela 7, avaliando as variedades, observa-se que a variedade Campinas (variedade 1) foi a que apresentou melhor desempenho, apresentando maior produtividade e maior número de frutos, fato também observado nos ambientes 1 e 2 .

Nas Tabelas 8 e 9, estão apresentados os testes de Tukey para comparação de médias dos desdobramentos das interações, no ambiente 3 , em nível de $5 \%$ de probabilidade.

De acordo com a Tabela 8, onde está apresentado o desdobramento das variedades dentro dos sistemas de produção, observa-se que, para os sistemas 1 e 2, a variedade Campinas apresentou maior produtividade e número de frutos que as demais variedades. Para o sistema 3, as variedades Campinas e Tudla, que não diferiram significativamente da variedade Seascape, apresentaram maior produtividade que a variedade Sweet Charlie; no entanto, a variedade Campinas apresentou maior número de frutos que as demais variedades. Já para o sistema 4, a variedade Seascape, que não diferiu significativamente da variedade Campinas, apresentou maior produtividade que as variedades Sweet Charlie e Tudla, apresentando, também, maior número de frutos.

Analisando o desdobramento de sistemas dentro das variedades, (Tabela 9), avaliando a média de produtividade e de número de frutos dos sistemas dentro de cada variedade, separadamente, observa-se que, para todas as variedades, o sistema de $150 \mathrm{~mm}$ com vaso contendo fibra de coco (sistema 3 ) apresentou o melhor desenvolvimento das plantas, tanto para a produtividade como para o número de frutos.

TABELA 1 - Teste de Tukey da Produtividade em gramas (P) e do número de frutos, para as parcelas (sistemas - S) e subparcelas (variedades - V) no ambiente 1, e os coeficientes de variação $(\mathrm{CV})$.

\begin{tabular}{|c|c|c|c|c|c|c|c|c|c|}
\hline \multicolumn{5}{|c|}{ Sistemas } & \multicolumn{5}{|c|}{ Variedades } \\
\hline $\mathrm{S}$ & $\mathrm{P}$ & & $\mathrm{S}$ & $\mathrm{NF}$ & $\mathrm{V}$ & $\mathrm{P}$ & $\mathrm{V}$ & $\mathrm{NF}$ & \\
\hline 3 & $329,37 A$ & A & 3 & $49,40 \mathrm{~A}$ & 1 & $293,09 \mathrm{~A}$ & 1 & $51,85 A$ & \\
\hline 2 & 169,39 & B & 2 & $26,00 \quad \mathrm{~B}$ & 4 & 194,68 B & 3 & 26,65 & B \\
\hline 1 & 164,77 & B & 1 & $23,25 \quad \mathrm{~B}$ & 3 & $170,42 \quad \mathrm{BC}$ & 2 & 21,20 & $\mathrm{C}$ \\
\hline 4 & 129,94 & $\mathrm{C}$ & 4 & $21,50 \quad \mathrm{~B}$ & 2 & $135,28 \quad \mathrm{C}$ & 4 & 20,45 & $\mathrm{C}$ \\
\hline CV (\%) & 17,55 & & CV(\%) & 19,51 & $\mathrm{CV}(\%)$ & 21,99 & $\mathrm{CV}(\%)$ & 19,38 & \\
\hline
\end{tabular}

* Médias seguidas de mesma letra, na coluna, não diferem entre si, pelo teste de Tukey, a $5 \%$ de probabilidade.

TABELA 2 - Teste de Tukey para o desdobramento das variedades (V) dentro dos sistemas (S), para a produtividade (P) e para o número de frutos (NF), no ambiente 1.

\begin{tabular}{|c|c|c|c|c|c|c|c|c|c|c|c|}
\hline \multicolumn{3}{|c|}{ Sistema 1} & \multicolumn{3}{|c|}{ Sistema 2} & \multicolumn{3}{|c|}{ Sistema 3} & \multicolumn{3}{|c|}{ Sistema 4} \\
\hline $\mathrm{V}$ & $\mathrm{P}$ & & $\mathrm{V}$ & $\mathrm{P}$ & & $\mathrm{V}$ & $\mathrm{P}$ & & $\mathrm{V}$ & $\mathrm{P}$ & \\
\hline 1 & 264,31 & A & 1 & 289,53 & A & 1 & 416,46 & A & 1 & 202,03 & A \\
\hline 4 & 222,73 & A & 4 & 182,29 & B & 4 & 320,72 & B & 2 & 140,20 & $\mathrm{AB}$ \\
\hline 3 & 108,07 & B & 3 & 149,16 & B & 3 & 299,92 & B & 3 & 124,55 & $\mathrm{BC}$ \\
\hline 2 & 63,95 & B & 2 & 56,59 & $\mathrm{C}$ & 2 & 280,39 & B & 4 & 52,96 & $\mathrm{C}$ \\
\hline $\mathrm{V}$ & $\mathrm{NF}$ & & $\mathrm{V}$ & $\mathrm{NF}$ & & $\mathrm{V}$ & $\mathrm{NF}$ & & $\mathrm{V}$ & $\mathrm{NF}$ & \\
\hline 1 & 44,60 & A & 1 & 51,20 & A & 1 & 75,20 & A & 1 & 36,40 & A \\
\hline 4 & 21,60 & B & 3 & 26,20 & B & 2 & 44,40 & B & 2 & 23,20 & B \\
\hline 3 & 18,20 & $\mathrm{BC}$ & 4 & 18,00 & $\mathrm{BC}$ & 3 & 43,40 & B & 3 & 18,80 & B \\
\hline 2 & 8,60 & $\mathrm{C}$ & 2 & 8,60 & $\mathrm{C}$ & 4 & 34,60 & B & 4 & 7,60 & $\mathrm{C}$ \\
\hline
\end{tabular}

* Médias seguidas de mesma letra, na coluna, não diferem entre si, pelo teste de Tukey, a 5\% de probabilidade. 
TABELA 3 - Teste de Tukey para o desdobramento dos sistemas (S) dentro das variedades (V), para a produtividade (P) e para o número de frutos (NF), no ambiente 1.

\begin{tabular}{|c|c|c|c|c|c|c|c|c|}
\hline \multicolumn{2}{|c|}{ Variedade 1} & \multicolumn{2}{|c|}{ Variedade 2} & \multicolumn{2}{|c|}{ Variedade 3} & \multicolumn{3}{|c|}{ Variedade 4} \\
\hline $\mathrm{S}$ & $\mathrm{P}$ & $\mathrm{S}$ & $\mathrm{P}$ & $\mathrm{S}$ & $\mathrm{P}$ & $\mathrm{S}$ & $\mathrm{P}$ & \\
\hline 3 & $416,46 \mathrm{~A}$ & 3 & $280,39 \mathrm{~A}$ & 3 & $299,92 \mathrm{~A}$ & 3 & 320,72 & A \\
\hline 2 & $289,53 \quad$ B & 4 & $140,20 \quad B$ & 2 & 149,16 В & 1 & 222,73 & B \\
\hline 1 & $264,31 \quad \mathrm{BC}$ & 1 & $63,95 \quad \mathrm{C}$ & 4 & $124,55 \quad$ B & 2 & 182,29 & B \\
\hline 4 & $202,03 \quad \mathrm{C}$ & 2 & $56,59 \quad \mathrm{C}$ & 1 & $108,07 \quad \mathrm{~B}$ & 4 & 52,96 & $\mathrm{C}$ \\
\hline $\mathrm{S}$ & NF & $\mathrm{S}$ & $\mathrm{NF}$ & $\mathrm{S}$ & $\mathrm{NF}$ & $\mathrm{S}$ & NF & \\
\hline 3 & $75,20 \mathrm{~A}$ & 3 & $44,40 \mathrm{~A}$ & 3 & $43,40 \mathrm{~A}$ & 3 & 34,60 & A \\
\hline 2 & $51,20 \quad \mathrm{~B}$ & 4 & $23,20 \quad \mathrm{~B}$ & 2 & $26,20 \quad \mathrm{~B}$ & 1 & 21,60 & B \\
\hline 1 & $44,60 \quad \mathrm{BC}$ & 1 & $8,60 \quad \mathrm{C}$ & 4 & $18,80 \quad \mathrm{~B}$ & 2 & 18,00 & B \\
\hline 4 & $36,40 \quad \mathrm{C}$ & 2 & $8,60 \quad \mathrm{C}$ & 1 & $18,20 \quad \mathrm{~B}$ & 4 & 7,60 & $\mathrm{C}$ \\
\hline
\end{tabular}

* Médias seguidas de mesma letra, na coluna, não diferem entre si, pelo teste de Tukey, a 5\% de probabilidade.

TABELA 4 - Teste de Tukey da Produtividade em gramas (P) e do número de frutos, para as parcelas (sistemas - S) e subparcelas (variedades - V) no ambiente 2, e os coeficientes de variação (CV).

\begin{tabular}{|c|c|c|c|c|c|c|c|}
\hline \multicolumn{4}{|c|}{ Sistemas } & \multicolumn{4}{|c|}{ Variedades } \\
\hline $\mathrm{S}$ & $\mathrm{P}$ & $\mathrm{S}$ & $\mathrm{NF}$ & V & $\mathrm{P}$ & $\mathrm{V}$ & $\mathrm{NF}$ \\
\hline 3 & $358,63 \mathrm{~A}$ & 3 & $52,60 \mathrm{~A}$ & 1 & $291,32 \mathrm{~A}$ & 1 & $50,75 \mathrm{~A}$ \\
\hline 2 & $151,23 \quad$ B & 4 & $22,95 \quad \mathrm{~B}$ & 4 & $188,70 \quad$ B & 3 & $24,35 \quad$ B \\
\hline 4 & $140,76 \quad \mathrm{~B}$ & 1 & $21,60 \quad \mathrm{~B}$ & 3 & $153,53 \quad$ B & 2 & $22,40 \quad \mathrm{~B}$ \\
\hline 1 & $135,52 \quad \mathrm{~B}$ & 2 & $21,60 \quad \mathrm{~B}$ & 2 & 152,59 В & 4 & $21,25 \quad \mathrm{~B}$ \\
\hline CV(\%) & 20,96 & $\mathrm{CV}(\%)$ & 25,98 & CV(\%) & 29,11 & $\mathrm{CV}(\%)$ & 28,53 \\
\hline
\end{tabular}

* Médias seguidas de mesma letra, na coluna, não diferem entre si, pelo teste de Tukey, a 5\% de probabilidade.

TABELA 5 - Teste de Tukey para o desdobramento das variedades (V) dentro dos sistemas (S), para a produtividade (P) e para o número de frutos $(\mathrm{NF})$, no ambiente 2.

\begin{tabular}{|c|c|c|c|c|c|c|c|c|c|}
\hline \multicolumn{3}{|c|}{ Sistema 1} & \multicolumn{3}{|c|}{ Sistema 2} & \multicolumn{2}{|c|}{ Sistema 3} & \multicolumn{2}{|c|}{ Sistema 4} \\
\hline V & $\mathrm{P}$ & & $\mathrm{V}$ & $\mathrm{P}$ & & $\mathrm{V}$ & $\mathrm{P}$ & $\mathrm{V}$ & $\mathrm{P}$ \\
\hline 1 & 285,19 & A & 1 & $267,62 A$ & A & 1 & $445,88 \mathrm{~A}$ & 3 & $179,06 \mathrm{~A}$ \\
\hline 4 & 122,83 & B & 4 & 164,03 & B & 4 & $406,79 \mathrm{~A}$ & 1 & $166,58 \mathrm{~A}$ \\
\hline 3 & 77,45 & $\mathrm{~B}$ & 2 & $91,93 \quad \mathrm{~F}$ & B & 2 & $305,58 \quad \mathrm{~B}$ & 2 & $156,26 \mathrm{AB}$ \\
\hline 2 & 56,60 & $\mathrm{~B}$ & 3 & $81,33 \quad \mathrm{I}$ & $\mathrm{B}$ & 3 & $276,26 \quad \mathrm{~B}$ & 4 & 61,14 B \\
\hline $\mathrm{V}$ & NF & & $\mathrm{V}$ & $\mathrm{NF}$ & & $\mathrm{V}$ & $\mathrm{NF}$ & $\mathrm{V}$ & $\mathrm{NF}$ \\
\hline 1 & 52,60 & A & 1 & $42,80 \mathrm{~A}$ & A & 1 & $76,00 \mathrm{~A}$ & 1 & $31,60 \mathrm{~A}$ \\
\hline 4 & 13,00 & $\mathrm{~B}$ & 3 & $15,40 \quad \mathrm{~B}$ & B & 4 & $48,00 \quad \mathrm{~B}$ & 3 & $28,20 \mathrm{~A}$ \\
\hline 3 & 11,80 & $\mathrm{~B}$ & 4 & $15,00 \quad \mathrm{~B}$ & B & 2 & $44,40 \quad \mathrm{~B}$ & 2 & $23,00 \mathrm{AB}$ \\
\hline 2 & 9,00 & $\mathrm{~B}$ & 2 & $13,20 \quad \mathrm{~B}$ & B & 3 & $42,00 \quad \mathrm{~B}$ & 4 & $9,00 \quad \mathrm{~B}$ \\
\hline
\end{tabular}

* Médias seguidas de mesma letra, na coluna, não diferem entre si, pelo teste de Tukey, a $5 \%$ de probabilidade.

TABELA 6 - Teste de Tukey para o desdobramento dos sistemas (S) dentro das variedades (V), para a produtividade (P) e para o número de frutos (NF), no ambiente 2 .

\begin{tabular}{|c|c|c|c|c|c|c|c|c|}
\hline \multicolumn{2}{|c|}{ Variedade 1} & \multicolumn{2}{|c|}{ Variedade 2} & \multicolumn{2}{|c|}{ Variedade 3} & \multicolumn{3}{|c|}{ Variedade 4} \\
\hline $\mathrm{S}$ & $\mathrm{P}$ & $\mathrm{S}$ & $\mathrm{P}$ & $\mathrm{S}$ & $\mathrm{P}$ & $\mathrm{S}$ & $\mathrm{P}$ & \\
\hline 3 & $445,88 \mathrm{~A}$ & 3 & $305,58 \mathrm{~A}$ & 3 & $276,26 \mathrm{~A}$ & 3 & 406,79 & A \\
\hline 1 & 285,19 B & 4 & $156,26 \quad$ В & 4 & $179,06 \quad \mathrm{~B}$ & 2 & 164,03 & B \\
\hline 2 & 267,62 B & 2 & $91,93 \quad \mathrm{BC}$ & 2 & 81,33 & 1 & 122,83 & $\mathrm{BC}$ \\
\hline 4 & 166,58 & 1 & $56,60 \quad \mathrm{C}$ & 1 & 77,45 & 4 & 61,14 & $\mathrm{C}$ \\
\hline $\mathrm{S}$ & $\mathrm{NF}$ & $\mathrm{S}$ & $\mathrm{NF}$ & $\mathrm{S}$ & $\mathrm{NF}$ & $\mathrm{S}$ & $\mathrm{NF}$ & \\
\hline 3 & $76,00 \mathrm{~A}$ & 3 & $44,40 \mathrm{~A}$ & 3 & $42,00 \mathrm{~A}$ & 3 & 48,00 & A \\
\hline 1 & $52,60 \quad \mathrm{~B}$ & 4 & $23,00 \quad \mathrm{~B}$ & 4 & $28,20 \mathrm{AB}$ & 2 & 15,00 & B \\
\hline 2 & $42,80 \quad \mathrm{BC}$ & 2 & $13,20 \quad \mathrm{BC}$ & 2 & $15,40 \quad \mathrm{BC}$ & 1 & 13,00 & B \\
\hline 4 & $31,60 \quad \mathrm{C}$ & 1 & 9,00 & 1 & 11,80 & 4 & 9,00 & B \\
\hline
\end{tabular}

* Médias seguidas de mesma letra, na coluna, não diferem entre si, pelo teste de Tukey, a 5\% de probabilidade. 
TABELA 7 - Teste de Tukey da Produtividade em gramas (P) e do número de frutos, para as parcelas (sistemas - S) e subparcelas (variedades - V) no ambiente 3 , e os coeficientes de variação (CV).

\begin{tabular}{|c|c|c|c|c|c|c|c|}
\hline \multicolumn{4}{|c|}{ Sistemas } & \multicolumn{4}{|c|}{ Variedades } \\
\hline $\mathrm{S}$ & $\mathrm{P}$ & $\mathrm{S}$ & $\mathrm{NF}$ & $\mathrm{V}$ & $\mathrm{P}$ & $\mathrm{V}$ & $\mathrm{NF}$ \\
\hline 3 & $680,09 \mathrm{~A}$ & 3 & $102,60 \mathrm{~A}$ & 1 & $549,54 \mathrm{~A}$ & 1 & $103,00 \mathrm{~A}$ \\
\hline 2 & $349,44 \quad$ B & 2 & $52,10 \quad$ B & 2 & $379,37 \quad$ B & 2 & $54,40 \quad \mathrm{~B}$ \\
\hline 4 & 315,02 B & 4 & $49,65 \quad \mathrm{~B}$ & 4 & 374,12 B & 3 & $50,25 \quad \mathrm{BC}$ \\
\hline 1 & 298,94 B & 1 & $44,00 \quad \mathrm{~B}$ & 3 & 340,45 B & 4 & $40,70 \quad C$ \\
\hline CV(\%) & 18,28 & $\mathrm{CV}(\%)$ & 20,45 & $\mathrm{CV}(\%)$ & 20,38 & $\mathrm{CV}^{(\%)}$ & 20,65 \\
\hline
\end{tabular}

* Médias seguidas de mesma letra, na coluna, não diferem entre si, pelo teste de Tukey, a 5\% de probabilidade.

TABELA 8 - Teste de Tukey para o desdobramento das variedades (V) dentro dos sistemas (S), para a produtividade (P) e para o número de frutos (NF), no ambiente 3.

\begin{tabular}{|c|c|c|c|c|c|c|c|c|c|}
\hline \multicolumn{3}{|c|}{ Sistema 1} & \multicolumn{2}{|c|}{ Sistema 2} & \multicolumn{2}{|c|}{ Sistema 3} & \multicolumn{3}{|c|}{ Sistema 4} \\
\hline $\mathrm{V}$ & $\mathrm{P}$ & & $\mathrm{V}$ & $\mathbf{P}$ & $\mathrm{V}$ & $\mathrm{P}$ & $\mathrm{V}$ & $\mathrm{P}$ & \\
\hline 1 & 555,38 & A & 1 & $498,78 \mathrm{~A}$ & 1 & $797,26 \mathrm{~A}$ & 2 & 416,55 & A \\
\hline 4 & 279,27 & B & 3 & $357,43 \quad$ B & 4 & $717,07 \mathrm{~A}$ & 1 & 346,76 & AB \\
\hline 3 & 196,12 & B & 4 & $276,32 \quad$ B & 2 & $670,69 \mathrm{AB}$ & 3 & 272,92 & B \\
\hline 2 & 165,01 & B & 2 & $265,24 \quad \mathrm{~B}$ & 3 & $535,34 \quad$ В & 4 & 223,83 & B \\
\hline $\mathrm{V}$ & NF & & $\mathrm{V}$ & NF & $\mathrm{V}$ & NF & $\mathrm{V}$ & NF & \\
\hline 1 & 96,60 & A & 1 & $98,60 \mathrm{~A}$ & 1 & $153,00 \mathrm{~A}$ & 2 & 65,20 & A \\
\hline 3 & 30,80 & B & 3 & $51,60 \mathrm{~B}$ & 2 & $100,40 \quad$ B & 1 & 63,80 & A \\
\hline 4 & 27,20 & B & 2 & $30,60 \quad \mathrm{BC}$ & 4 & $80,20 \mathrm{BC}$ & 3 & 41,80 & B \\
\hline 2 & 21,40 & B & 4 & 27,60 & 3 & 76,80 & 4 & 27,80 & B \\
\hline
\end{tabular}

* Médias seguidas de mesma letra, na coluna, não diferem entre si pelo teste de Tukey, a 5\% de probabilidade.

TABELA 9 - Teste de Tukey para o desdobramento dos sistemas (S) dentro das variedades (V), para a produtividade (P) e para o número de frutos (NF), no ambiente 3.

\begin{tabular}{|c|c|c|c|c|c|c|c|c|}
\hline \multicolumn{2}{|c|}{ Variedade 1} & \multicolumn{2}{|c|}{ Variedade 2} & \multicolumn{2}{|c|}{ Variedade 3} & \multicolumn{3}{|c|}{ Variedade 4} \\
\hline $\mathrm{S}$ & $\mathrm{P}$ & $\mathrm{S}$ & $\mathrm{P}$ & $\mathrm{S}$ & $\mathrm{P}$ & $\mathrm{S}$ & $\mathrm{P}$ & \\
\hline 3 & $797,26 \mathrm{~A}$ & 3 & $670,69 \mathrm{~A}$ & 3 & $535,34 \mathrm{~A}$ & 3 & 717,07 & A \\
\hline 1 & $555,38 \quad \mathrm{~B}$ & 4 & $416,55 \mathrm{~B}$ & 2 & 357,43 B & 1 & 279,27 & B \\
\hline 2 & $498,78 \quad \mathrm{~B}$ & 2 & $265,24 \quad C$ & 4 & $272,92 \mathrm{BC}$ & 2 & 276,32 & $\mathrm{~B}$ \\
\hline 4 & $346,76 \quad \mathrm{C}$ & 1 & $165,01 \quad \mathrm{C}$ & 1 & $196,11 \quad \mathrm{C}$ & 4 & 223,83 & B \\
\hline $\mathrm{S}$ & $\mathrm{NF}$ & $\mathrm{S}$ & $\mathrm{NF}$ & $\mathrm{S}$ & $\mathrm{NF}$ & $\mathrm{S}$ & $\mathrm{NF}$ & \\
\hline 3 & $153,00 \mathrm{~A}$ & 3 & $100,40 \mathrm{~A}$ & 3 & $76,80 \mathrm{~A}$ & 3 & 80,20 & A \\
\hline 2 & $98,60 \quad \mathrm{~B}$ & 4 & $65,20 \quad B$ & 2 & $51,60 \quad \mathrm{~B}$ & 4 & 27,80 & B \\
\hline 1 & $96,60 \quad \mathrm{~B}$ & 2 & $30,60 \quad \mathrm{C}$ & 4 & $41,80 \quad \mathrm{~B}$ & 2 & 27,60 & $\mathrm{~B}$ \\
\hline 4 & $63,80 \quad \mathrm{C}$ & 1 & $21,40 \quad \mathrm{C}$ & 1 & $30,80 \quad \mathrm{~B}$ & 1 & 27,20 & $\mathrm{~B}$ \\
\hline
\end{tabular}

* Médias seguidas de mesma letra, na coluna, não diferem entre si, pelo teste de Tukey, a 5\% de probabilidade.

\section{CONCLUSÕES}

Depois de analisar todos os efeitos principais e suas interações, pode-se concluir que:

1-O melhor sistema de cultivo foi o de canais de $150 \mathrm{~mm}$ com vaso contendo fibra de coco, em todos os ambientes estudados.

2- A variedade Campinas foi a que apresentou melhor desenvolvimento, em todos os ambientes analisados.

3- Para o sistema de canais de $100 \mathrm{~mm}$ em NFT, no ambiente 1, e para o sistema de canais de $150 \mathrm{~mm}$ com vaso contendo fibra de coco, nos ambientes 2 e 3, a variedade Tudla apresentou produtividade similar à da variedade Campinas.

4- Para o sistema de tubos verticais, nos ambientes 1 e 3 , a variedade Seascape apresentou produtividade similar à da variedade Campinas, e, no ambiente 2, a variedade Sweet Charlie foi similar à variedade Campinas.
5- As variedades Campinas e Tudla foram menos produtivas no sistema de tubos verticais, nos três ambientes.

6- As variedades Seascape e Sweet Charlie foram as menos produtivas no sistema de canais de $100 \mathrm{~mm}$ em NFT, nos três ambientes.

\section{AGRADECIMENTOS}

Os autores agradecem à FAPESP (Fundação de Amparo à Pesquisa do Estado de São Paulo), pelos recursos de auxílio à pesquisa concedidos (Processo $n^{\circ}$ 99/04753-4), e à CAPES (Coordenação de Aperfeiçoamento de Pessoal de Nível Superior), pela bolsa de Doutorado, tornando possível a realização deste trabalho científico. 


\section{REFERÊNCIAS}

BERNARDI, J.; HOFFMANN, A.; ANTUNES, L. E. C.; FREIRE, J. M. Cultivares. In: SANHUEZA, R.M.V.; HOFFMANN, H.; ANTUNES, L.E.C.; FREIRE J.M. Sistema de produção de morango para mesa na região da serra gaúcha e encosta superior do Nordeste. Bento Gonçalves: Embrapa Uva e Vinho. (Sistema de Produção, 6). Disponível em: <http://sistemasdeproducao.cnptia.embrapa.br/ FontesHTML/Morango/MesaSerraGaucha/cultivares.htm>. Acesso em: 10 out 2007.

CARRIJO, O. A. et al. Tomato crop production under different substrates and greenhouse models. Horticultura Brasileira, Brasília, v. 22, n. 1, p.5-9, 2004.

FERNANDES JÚNIOR, F. Produção do morangueiro em solo, hidroponia NFT e colunas verticais com substrato. 2001. 88f. Dissertação (Mestrado ) - Instituto agronômico de Campinas, Campinas, 2001.

GOTO, R.; TIVELLI, S. B. Produção de hortaliças em ambiente protegido: condições subtropicais. São Paulo: Fundação Editora da Unesp, 1998. 319 p.

GUSMÃO, M. T. A. Análise do comportamento da cultura do morangueiro (Fragaria $x$ ananassa Duch.) em condições de cultivo hidropônico. 2000. 60 f. Dissertação (Mestrado em Agronomia/Produção Vegetal) - Faculdade de Ciências Agrárias e Veterinárias, Universidade Estadual Paulista, 2000.
NUNES, M. U. C. Produção de mudas de hortaliças com o uso da plasticultura e do pó de coco. Aracaju: Embrapa Tabuleiros Costeiros, 2000.29p. (Circular Técnica 13).

OLIVEIRA, R. P.; NINO, A. F. P.; SILVA, F. O. X.; BRAHM, R. U. Produção e obtenção de mudas. In: GOMES, J.C.C. Produção de matrizes de morangueiro por meio de cultura de tecidos. Pelotas: Embrapa Clima Temperado, 2005. (Sistemas de Produção, 7). Disponível em: <http://sistemasdeproducao.cnptia.embrapa.br/ FontesHTML/Morango/MatrizesMorangueiro/cap05.htm>. Acesso: 10 out. 2007.

PIRES, R. C. M.; PASSOS, F. A.; TANAKA, M. A. S. Irrigação do morangueiro. Informe Agropecuário, Belo Horizonte, v. 20, n. 198, p. 52-58, 1999.

TAVARES, H. L. Cultivo de morangueiro (Fragaria $\mathbf{x}$ ananassa Duch.) em sistema hidropônico no Distrito Federal. 2001. 183 f. Dissertação (Mestrado em Ciências Agrárias) - Universidade de Brasília, Brasília, 2001

UNIVERSIDADEESTADUALPAULISTA“JÚLIODEMESQUITA FILHO”. Departamento de Ciências Exatas. ESTAT. Versão 2.0. Jaboticabal: FCAV/UNESP, 1994.

VILLELA JUNIOR, L.V. E.; ARAUJO, J. A. C.; FACTOR, T. L. Análise do resfriamento da solução nutritiva para o cultivo hidropônico do morangueiro. Engenharia Agrícola, Jaboticabal, v. 24, n. 2, p. 338-346, 2004. 